\title{
Antiviral Combination Clinically Better Than Standard Therapy in Severe but Not in Non-Severe COVID-19
}

\author{
Prasan Kumar Panda (D) \\ Budha O Singh' \\ Bikram Moirangthem' \\ Yogesh Arvind Bahurupi ${ }^{2}$ \\ Sarama Saha ${ }^{3}$ \\ Girraj Saini ${ }^{4}$ \\ Minakshi Dhar' \\ Mukesh Bairwa' \\ Venkatesh Srinivasa Pai ${ }^{1}$ \\ Ankit Agarwal ${ }^{5}$ \\ Girish Sindhwani ${ }^{6}$ \\ Shailendra Handu ${ }^{7}$ \\ Ravi Kant ${ }^{8}$ \\ 'Department of Medicine (Infectious
} Disease Division), All India Institute of Medical Sciences (AlIMS), Rishikesh, India; ${ }^{2}$ Department of Community and Family Medicine, All India Institute of Medical Sciences (AIIMS), Rishikesh, India; ${ }^{3}$ Department of Biochemistry, All India Institute of Medical Sciences (AllMS), Rishikesh, India; ${ }^{4}$ Department of Nursing, All India Institute of Medical Sciences (AIIMS), Rishikesh, India; ${ }^{5}$ Department of Critical Care Medicine, All India Institute of Medical Sciences (AlIMS), Rishikesh, India; ${ }^{6}$ Department of Pulmonary Medicine, All India Institute of Medical Sciences (AIIMS), Rishikesh, India; ${ }^{7}$ Department of Pharmacology, All India Institute of Medical Sciences (AllMS), Rishikesh, India; ${ }^{8}$ Department of General Surgery, All India Institute of Medical Sciences (AlIMS), Rishikesh, India

Correspondence: Prasan Kumar Panda Department of Medicine (Infectious Disease Division), All India Institute of Medical Sciences (AIIMS), Sixth Floor,

College Block, Rishikesh, 249203, India

Tel +9l 9868999488

Email motherprasanna@rediffmail.com
Purpose: Definitive antiviral treatment is not available for COVID-19 infection, with the exception of remdesivir, which still evokes many doubts. Various monotherapy or combination therapies with antivirals or other agents have been tried. The present study aims to evaluate the therapeutic potential of hydroxychloroquine and lopinavir-ritonavir in combination with ribavirin in mild-severe COVID-19.

Patients and Methods: A single-center, open-label, parallel-arm, stratified randomized controlled trial evaluated the therapeutic potential of combination antiviral therapies. Enrolled patients in the severe category were randomized into three groups: (A) standard treatment, (B) hydroxychloroquine+ribavirin+standard treatment, or (C) lopinavir+ritonavir +ribavirin+standard treatment; while the non-severe category comprised two groups: (A) standard treatment or (B) hydroxychloroquine+ribavirin. Combination antivirals were given for 10 days and followed for 28 days. The primary endpoints were safety, symptomatic and laboratory recovery of organ dysfunctions, and time to SARS-CoV-2 RT-PCR negative report.

Results: In total, 111 patients were randomized: 24, 23, and 24 in severe categories A, B, and $\mathrm{C}$, respectively, and 20 in each of the non-severe groups. Two patients receiving ribavirin experienced drug induced liver injury, and another developed QT prolongation after hydroxychloroquine. In the severe category, $47.6 \%, 55 \%$, and $30.09 \%$ in $\mathrm{A}, \mathrm{B}$, and $\mathrm{C}$ groups, respectively, showed symptomatic recovery, compared to $93.3 \%$ and $86.7 \%$ in $\mathrm{A}$ and B groups, respectively, in the non-severe category at 72 hours $(P>0.05)$.

Conclusion: Though the results failed to show statistical superiority of the antiviral combination therapies to that of the standard therapy in both the severe and non-severe categories in symptomatic adult patients of COVID-19 due to very small sized trial, clinically hydroxychloroquine+ribavirin therapy is showing better recovery by $7.4 \%$ than standard therapy in the former category. However, results do indicate the benefit of standard therapy in the non-severe category by $6.6 \%$. Furthermore, the dose of ribavirin needs to be reconsidered in the Indian population.

Keywords: coronavirus disease 2019, interventional trial, hydroxychloroquine, lopinavir/ ritonavir combination, ribavirin

\section{Introduction}

COVID-19 is an infectious disease caused by the novel coronavirus SARS-CoV-2. Individuals of all age groups and both sexes are at risk of infection, with a higher probability of severe disease in the elderly, pregnant, transplant recipients, and people with chronic medical conditions. ${ }^{1-3}$ The disease can progress from 
asymptomatic to mild cough, fever, and sore throat to acute respiratory distress syndrome, various other endorgan involvements like cardiac, dermatologic, hematological, hepatic, neurological, and renal, thromboembolic events, septic shock, or death, or even long COVID. ${ }^{4-9}$ These presentations can be categorized as non-severe and severe COVID-19 during disease progression from the viremia phase to the immunological phase.

Antiviral therapies are expected to have a higher benefit if administered early in the course with immunosuppressive or anti-inflammatory therapies which are likely to be beneficial in the later stages of COVID-19 infection. Supportive treatment forms the mainstay of non-severe cases of COVID-19 infections. Hydroxychloroquine (HCQ) is thought to be effective in treatment and prophylaxis. ${ }^{10,11}$ It has got immunomodulatory effects and it may also interfere with the binding of severe acute respiratory syndrome-associated coronavirus (SARS-CoV) to the cell receptor, thus inhibiting viral entry. ${ }^{12}$ There have been no significant adverse effects reported on HCQ at the doses in use for the treatment of COVID-19; however its known side-effects, such as QTc prolongation, hypoglycemia, neuropsychiatric effects, and retinopathy, warrant constant observation. ${ }^{13}$ The replication of severe acute respiratory syndrome coronavirus 2 (SARSCoV-2) depends on two proteases, viz. 3-chymotrypsin-like protease (3CLpro) and papain-like protease (PLpro), are responsible for cleaving polyproteins into an RNAdependent RNA polymerase and a helicase. Lopinavir-ritonavir combination has also been tried in hospitalized patients due to its probable mechanism of inhibiting these proteases. ${ }^{14,15}$

Ribavirin on the other hand has been shown to have direct antiviral activity in vitro against the SARS virus and any strain variants that may emerge. ${ }^{16}$ Apart from the COVID-19 viremia with the potential to cause liver injury, both lopinavir-ritonavir and ribavirin are known to cause transaminitis and, especially when given in combination, can raise concerns of exacerbating liver injury. ${ }^{17}$ Lopinavir-ritonavir in isolation also causes gastrointestinal side-effects such as nausea and diarrhoea. Ribavirin is contraindicated in pregnancy as it is a known teratogen. ${ }^{17}$

Numerous studies on these individual antivirals and HCQ have failed to establish any significant benefits. ${ }^{18}$ However, combination therapy of these has not been attempted.

Hence, this study aims to define the outcome with these agents in combination in comparison to that of the standard supportive therapy.

\section{Materials and Methods}

\section{Study Settings, Design, and Population}

The study was a single-center, open-label, parallel-arm, stratified randomized controlled trial conducted at a tertiary care center between June 2020 to May 2021 after obtaining approval of the Institutional Ethics Committee, All India Institute of Medical Sciences (AIIMS), Rishikesh. The trial was registered at the Clinical Trial Registry of India (CTRI/2020/06/025575).

Being an exploratory trial, in a relatively new infection, all consenting patients $\geq 18$ years old, diagnosed with symptomatic COVID-19 disease with a positive reverse-transcriptase-polymerase chain reaction (RTPCR) assay for SARS-CoV-2, were included in the trial. Exclusion criteria included: patients on medications which were contraindicated with lopinavir/ritonavir, hydroxychloroquine/chloroquine, or ribavirin; patients taking lopinavir-ritonavir based anti-retroviral therapy or on hydroxychloroquine/chloroquine or ribavirin; known allergic reactions to any of the drugs used in the treatment arms; the inability to take oral medications (lopinavir-ritonavir, hydroxychloroquine/chloroquine, ribavirin); pregnant or lactating females; patients who had received any of the experimental therapies for 2019nCoV (off-label, compassionate use, or trial-related) within 30 days before participation in the present study.

The enrolled patients were categorized as "severe disease" or "non-severe disease", based on pre-defined criteria (WHO classification of COVID-19 severity). "Severe disease" was defined as confirmed pneumonia on chest imaging, $\mathrm{SpO}_{2}<93 \%, \mathrm{PaO}_{2}: \mathrm{FiO}_{2}<300$, respiratory failure (need for mechanical ventilation), septic shock, multiple organ dysfunction syndromes, liver disease (Child-Pugh score $\geq \mathrm{C}$, AST $>5$ times upper limit), renal impairment (estimated glomerular filtration rate $\leq 30 \mathrm{~mL} / \mathrm{min} / 1.73 \mathrm{~m}^{2}$ ) or receiving renal replacement therapy (continuous renal replacement therapy, hemodialysis, or peritoneal dialysis), and other single organ failures (eg, heart, if specific definitions were met (NYHA classification for heart failure). The "non-severe disease" category included mild-to-moderate disease not fulfilling the criteria of severe disease.

Enrolled patients in the "severe disease" category were randomized dynamically into three arms: severe $\mathrm{A}$, severe B, severe C. Severe A received standard treatment as per institute protocol. Severe group B received a combination of $\mathrm{HCQ}+$ ribavirin+standard treatment. Severe group 
C received lopinavir+ritonavir+ribavirin+standard treatment; while the "non-severe disease" category patients were randomized into two arms: non-severe $\mathrm{A}$ and B. Non-severe A received the standard treatment and nonsevere $\mathrm{B}$ received $\mathrm{HCQ}+$ ribavirin+standard treatment.

\section{Randomization}

The randomization was done with a computer-based software "randomize $\mathrm{R}$ package" of version 1.4.2. Randomization was done in blocks of four and patients were stratified into non-severe and severe as per defined criteria of severity.

\section{Comparator}

In the severe category three parallel arms of interventions were compared while there were two in the non-severe category.

\section{Outcomes/Endpoints}

The primary endpoints were determination of safety, clinical recovery of symptoms, and laboratory recovery of each organ involvement, as evident from both clinical and laboratory parameters, respectively, and time to SARS-CoV-2 RT-PCR negative report of nasopharyngeal/ throat swab specimen from the day of the intervention. Safety was determined by observing the frequency and severity of serious adverse events as per the division of acquired immune deficiency syndrome (DAIDS) table for grading the severity of adverse events. Clinical recovery was defined as normalization of fever, respiratory rate, oxygen saturation, and alleviation of cough at first followup at 72 hours of initiation of therapy, all sustained for at least 72 hours. Recovery of the laboratory parameters (if deranged at presentation and attributed to the current infection and not due to ongoing other medical conditions) was also applied at 72 hour intervals. Normalization and alleviation criteria were: fever - axillary temperature $\leq 36.9^{\circ} \mathrm{C}$, oral temperature $\leq 37.2^{\circ} \mathrm{C}$, respiratory rate $\leq 24$ / minute on room air, $\mathrm{SpO}_{2}>94 \%$ on room air, mild or absent cough using a patient-reported ordinal scale (severe, moderate, mild, or absent).

The secondary endpoints included all-cause mortality (COVID-19 infection related/associated), respiratory progression (defined on any values of $\mathrm{SpO}_{2} \leq 94 \%$ on room air or in any values $\mathrm{PaO}_{2} / \mathrm{FiO}_{2}<300 \mathrm{mmHg}$ and requirement for supplemental oxygen or more advanced ventilator support such as non-invasive/invasive ventilation), and hospital duration of stay.
At the time of enrolment, demographic data were collected from the participants, including relevant data on their medical history, co-morbidities, as well as risk factors for severe COVID-19. Patients were assessed for clinical and laboratory improvements at 72 hours of initiation of treatment, repeated once every 72 hours until the primary endpoint was met. Investigations included complete blood counts, liver function tests, renal function tests, HbAlc (if a diagnosed diabetic or as per clinician's discretion if suspected), blood glucose, PT/INR, serum electrolytes, arterial blood gases, chest X-ray, ECG, other organ markers as per involvement, and RT-PCR for SARS-COV-2 (until negative on two occasions at least 24 hours apart). Patients who failed to respond to the current treatment arm were further managed as per the institute protocol, which incorporated the current treatment guidelines. All adverse events were recorded at the first instance including dates as appropriate. Each adverse event was assessed for severity, causality, seriousness, and expectedness. If any serious adverse event was recorded, the participant was withdrawn from the trial, and appropriate management done accordingly as per the institute's guidelines. Patients were followed up until 28 days from the day of enrolment.

\section{Statistical Analysis}

Data were described as mean $\pm \mathrm{SD}$ and proportions. Comparisons of the categorical variables of the arms were performed using Chi-square or Fisher's exact test. Participants enrolled in severe strata were analyzed using One-way ANOVA followed by a post-hoc test. An independent $t$-test was applied for the comparison of means between two arms in the non-severe strata. We followed per-protocol analysis for the entire study.

\section{Results}

In the study, a total of 550 patients were screened, of which 111 patients were enrolled after randomization (Figure 1). Seventy-one participants were enrolled in the severe category, of which 24, 23, and 24 participants were randomized into groups $\mathrm{A}, \mathrm{B}$, and $\mathrm{C}$, respectively. Forty participants were categorized into the non-severe category, 20 participants in each group A and B. Two participants: one from the severe B and one from non-severe B showed an adverse drug reaction in the form of elevated liver enzymes (after receiving 2.4gm ribavirin per oral stat followed by $1.2 \mathrm{gm}$ twice daily), which resolved after reduction of the doses to $1.2 \mathrm{gm}$ per oral stat followed by $0.6 \mathrm{gm}$ twice daily. One participant in the severe 


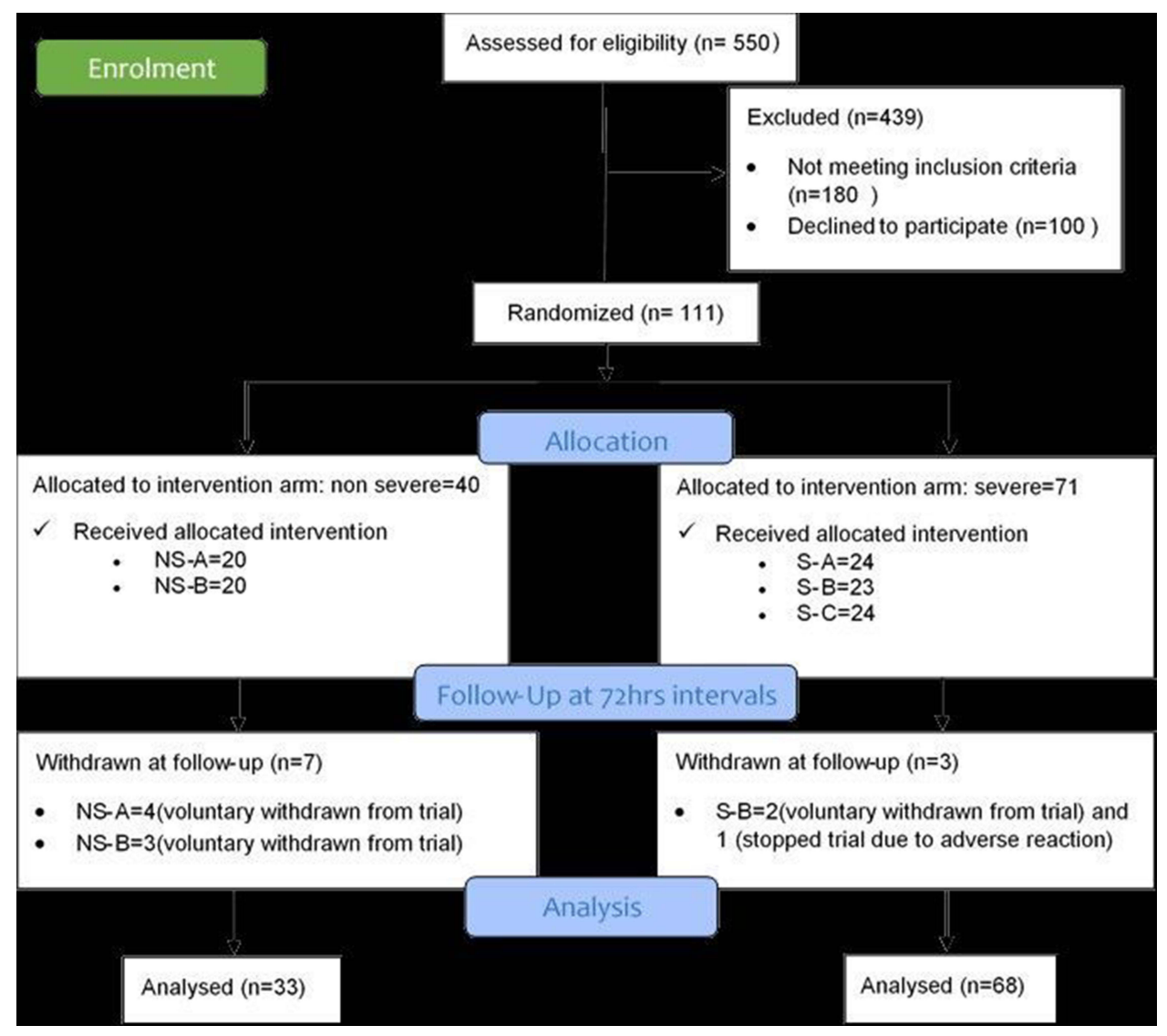

Figure I The study flow.

Abbreviations: NS, Non-severe; S, Severe category of intervention arms.

category B showed QT prolongation on the 3rd day of administration of HCQ and hence the offending drug was discontinued and the trial discontinued for the same patient. Four participants from the non-severe A, three from the non-severe $\mathrm{B}$, and two patients from the severe $\mathrm{B}$ had withdrawn from the trial.

Baseline characteristics: In the severe category, cough, shortness of breath, and fever were the primary reasons for participants to present in the health facility (Table 1). Diabetes mellitus, COPD, hypertension, and asthma were the comorbidities with which the participants presented, of which hypertension predominated. Similarly, in the non-severe category, participants presented with predominant complaints of fever, cough, and shortness of breath. Also, a trend of male predominance and older age groups were observed in all participants.

\section{Primary Outcome}

Except for two participants (one from severe B and another from the non-severe B) who developed elevated liver enzymes after high doses of ribavirin (dose reduced to half), and one participant who developed QT prolongation after day 3 of
HCQ (the offending agents were stopped), no other participants developed conditions that can be attributed to the trial drugs. The clinical recovery was assessed for presenting symptoms as the depicted trend of resolution of symptoms in the graph (Figure 2A-D). However, the difference in clinical recovery status was not statistically significant among three groups in the severe category and two groups in the non-severe category.

Concerning laboratory recovery such as organ function tests (hemogram, liver, and kidney function tests), all groups (both severe and non-severe category) showed similar non-significant increasing or decreasing trends concerning the time of admission and subsequent followups (Figure 3A-D).

The time to SARS-CoV-2 RT-PCR negative in nasopharyngeal swab specimen was also not significant among groups (Table 2).

\section{Secondary Outcome}

Analysis of secondary outcomes using the concurrent randomization analysis revealed that all-cause mortality was 


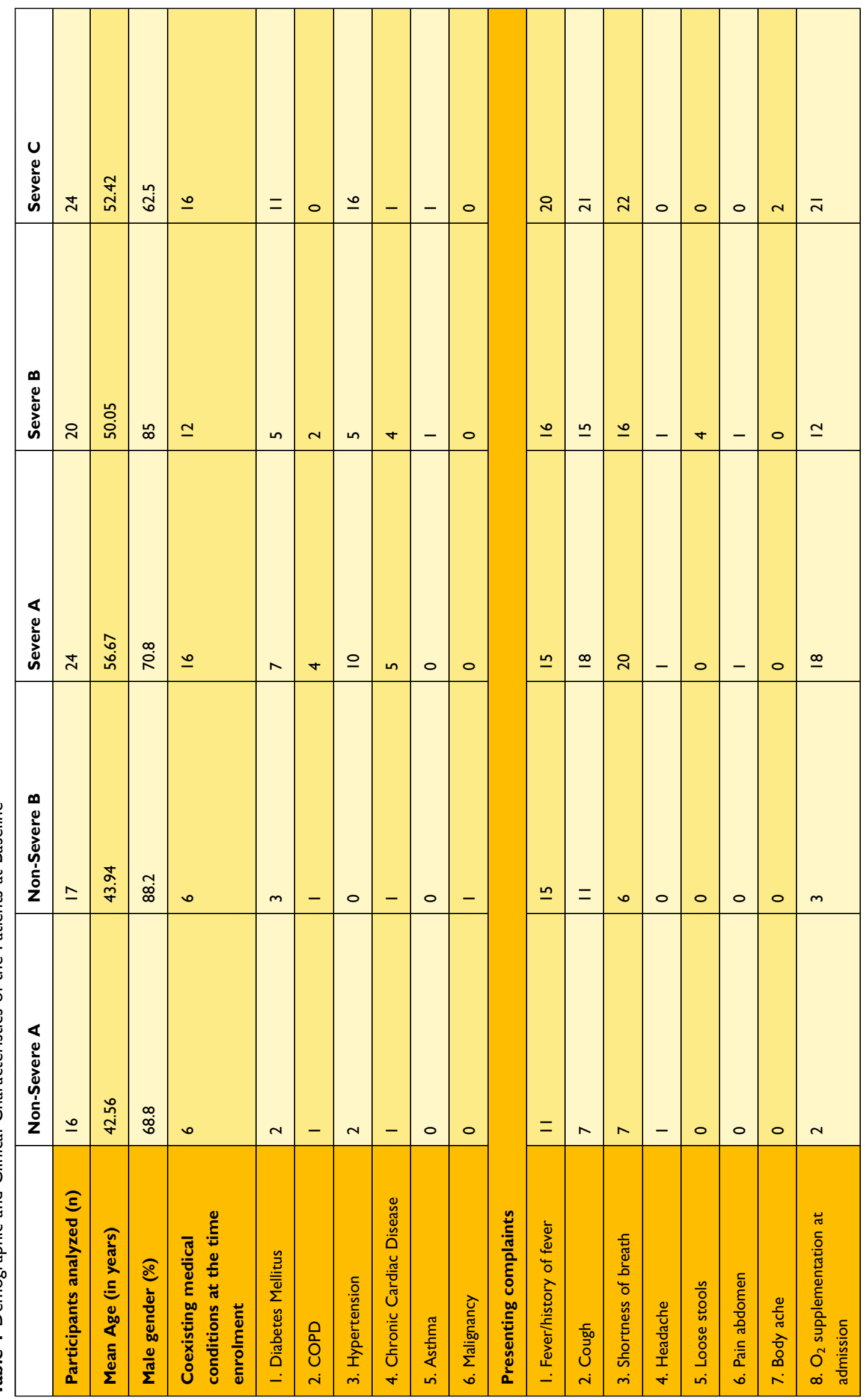




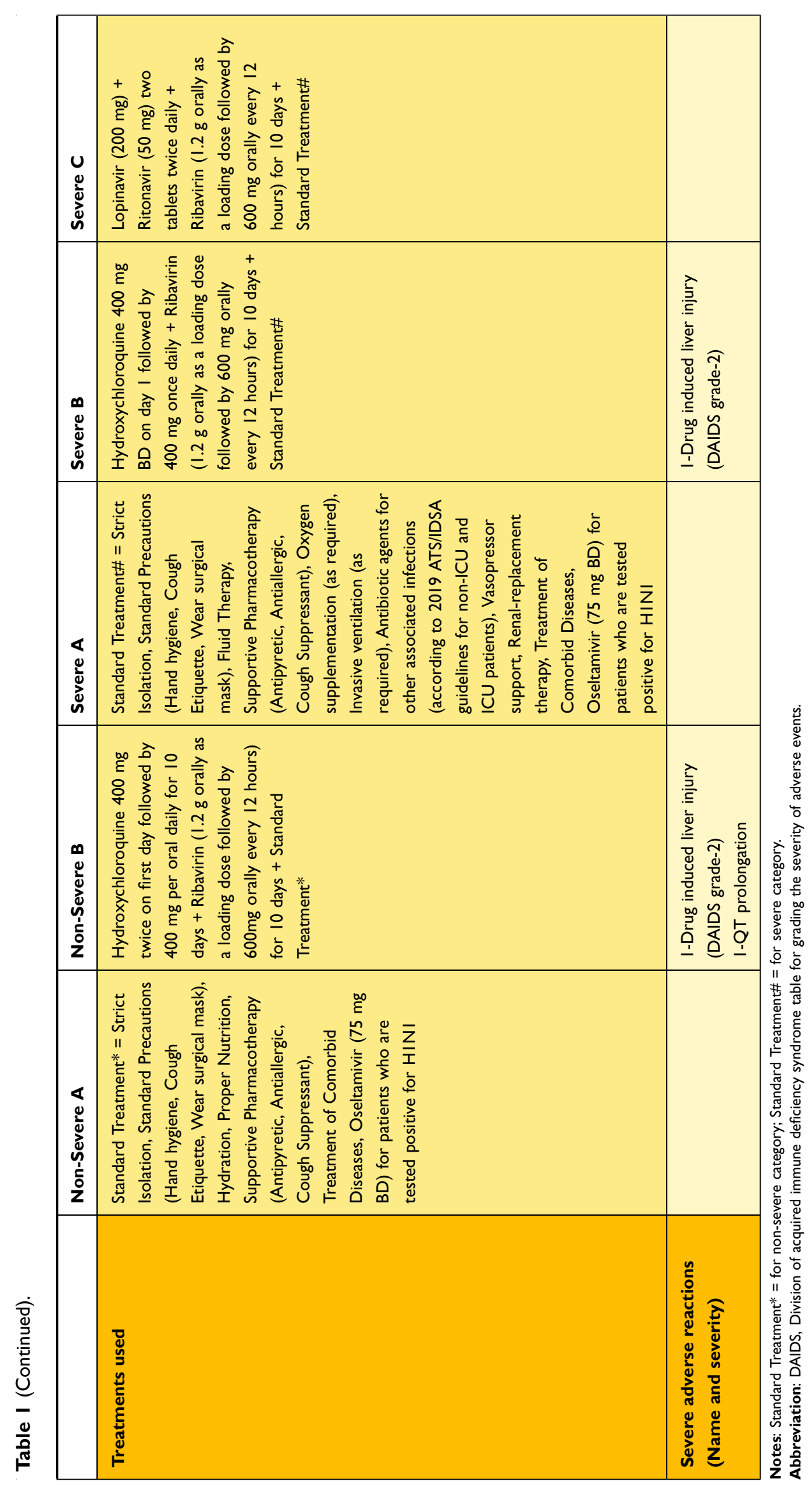



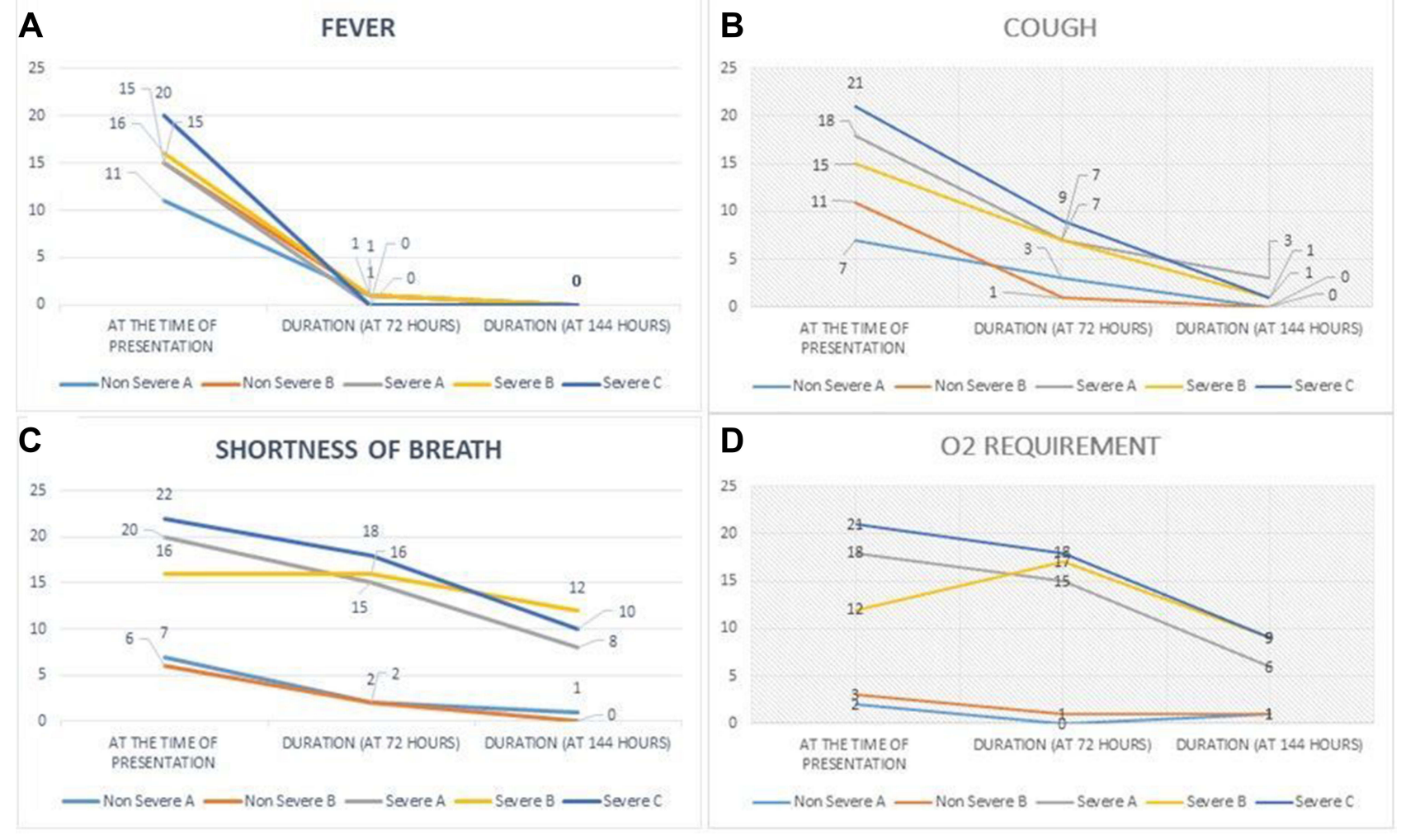

Figure 2 (A-D) Time trend of clinical recovery of major symptoms among different intervention arms.

lowest in category severe B followed by in category severe $\mathrm{C}$ and highest in category severe A without any intergroup statistical significance, and no mortality was recorded in the non-severe categories (Table 3). Similarly, we found no significant increase in the frequency of respiratory progression in the non-severe category, however, in severe categories, the need for positive pressure (both noninvasive and invasive) ventilation at admission gradually declined during consecutive follow-ups in all three groups, the least in severe group A, which, due to inadequate sample size, failed to reflect any statistically significant value. The average duration of hospital stays was 2 weeks for all severe groups.

In the case of the non-severe category, no significant finding could be made concerning the trend of increase in oxygen impairments. The duration of hospital stays remained 8.12 days $(\mathrm{SD}=3.20)$ in non-severe $\mathrm{A}$ and 10.18 days in non-severe $\mathrm{B}(\mathrm{SD}=6.347)$, respectively. Two participants from non-severe B had the intermittent requirement of NIV, which resolved and recovered in the subsequent follow-ups. One participant in the non-severe B had progression of the disease and was subsequently randomized to the severe groups, ultimately leading to the requirement of MV and hence discontinuation of the trial.
Two participants from the non-severe A and three participants from non-severe B were supplemented with low flow $\mathrm{O}_{2}$ because of background COPD. By the time of discharge, all participants in the non-severe groups had been weaned off from $\mathrm{O}_{2}$.

\section{Discussion}

This randomized controlled trial attempted to identify antiviral combination therapy for its potential benefits in the treatment of COVID-19 in both non-severe and severe manifestations of the same disease. Although with limited data having no statistically significant findings, clinical superiority of $7.4 \%$ could be observed in the severe category of COVID-19 by HCQ+ribavirin antiviral combination therapy and the standard therapy is more effective in the non-severe category by $6.6 \%$. The lopinavir+ritonavir +ribavirin combination is highly ineffective.

The degree of shortness of breath and other organ dysfunctions were the primary factors that guided in categorizing the severity of COVID-19 infections and this system held as only one patient in the non-severe group progressed to severe symptoms during the trial period, with the remaining participants in the category making an uneventful recovery. The predominance of the male sex group in all severities of the 

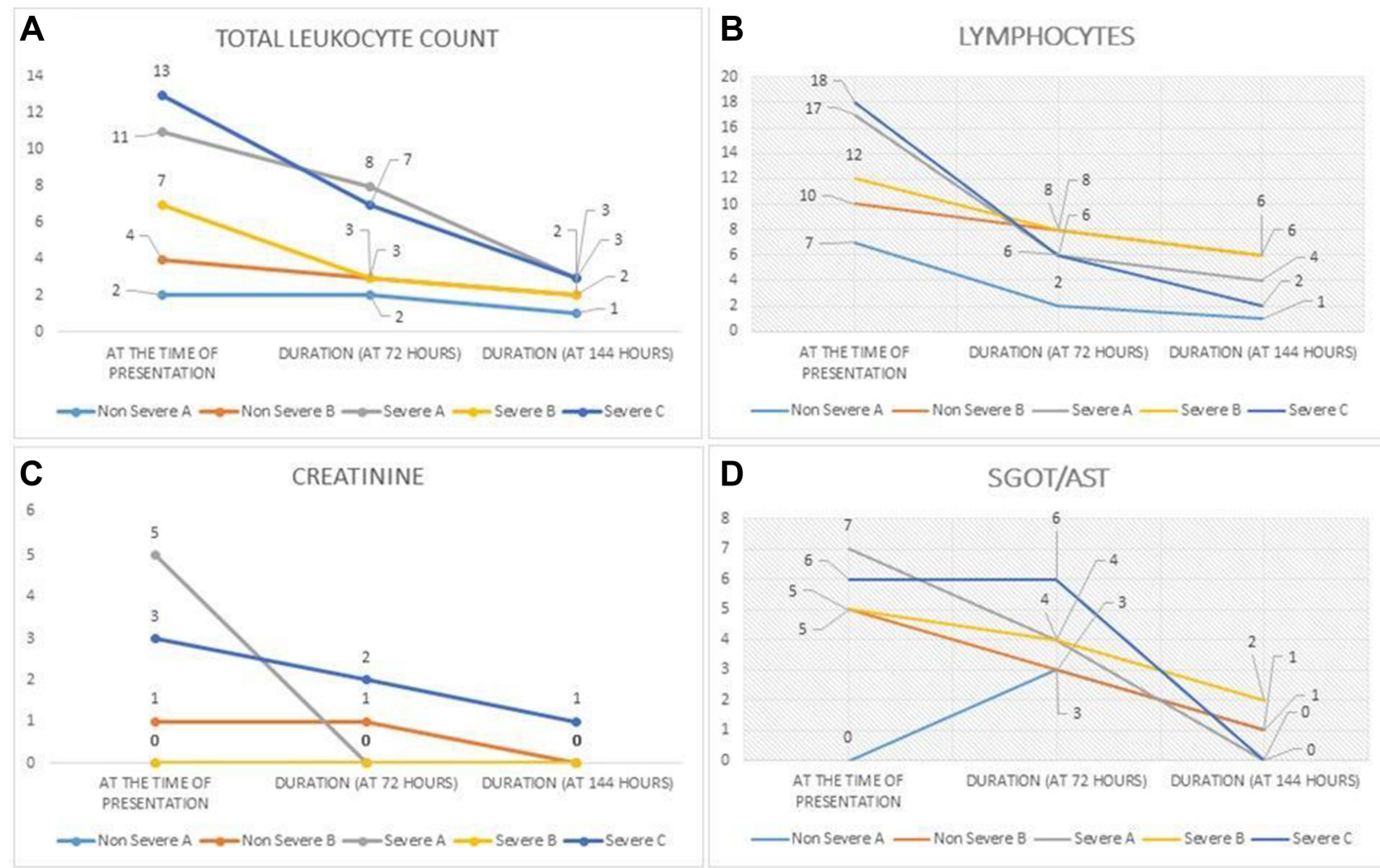

Figure 3 (A-D) Time trend of laboratory recovery of major tests among different intervention arms.

study groups is noteworthy. There are also additional findings of concomitant comorbid conditions, of which hypertension appears to be the most common among others. This may provide interest in future studies elaborating the intrinsic pathophysiology and disease outcomes concerning COVID19 among the hypertensives. ${ }^{19}$

In the comparative study of the non-severe category between the participants receiving standard treatment and HCQ+ribavirin, nearly all patients showed recovery during the study period and a clear association between the treatment outcomes of the two intervention groups could not be made. Hence, from the study, it can be concluded to treat mildmoderate (non-severe) disease with standard therapy, it is noteworthy to mention that almost all recover without any difference in the duration of illness or mortality. This may rationalize the use of scarce health care resources during this pandemic. $^{20}$

On the other hand, in the severe category, the study suggests that $\mathrm{HCQ}+$ ribavirin have recovered more and prevented the progression to more severe respiratory disease, as shown by the lower proportion of patients progressing to the need of NIV and MV compared to the standard treatment group without any statistical significance. At the same time, no significant side-effects were noted during the study period. Also, all-cause mortality is the lowest in severe group B. The number of samples may

Table 2 The Time to a Negative SARS-CoV-2 RT-PCR in Nasopharyngeal and Throat Swab Specimens

\begin{tabular}{|l|l|l|l|l|l|}
\hline & $\begin{array}{l}\text { Non-Severe } \\
\text { A }\end{array}$ & $\begin{array}{l}\text { Non-Severe } \\
\text { B }\end{array}$ & $\begin{array}{l}\text { Severe } \\
\text { A }\end{array}$ & $\begin{array}{l}\text { Severe } \\
\text { B }\end{array}$ & $\begin{array}{l}\text { Severe } \\
\text { C }\end{array}$ \\
\hline $\begin{array}{l}\text { No of participants having Negative RT-PCR at follow-up intervals until } \\
\text { discharge }\end{array}$ & 3 & 3 & 2 & 3 & 2 \\
\hline Mean duration (in days) & 5.66 & 6.33 & 12.5 & 8.5 & 8 \\
\hline P-value & Intergroup $>0.05$ (non-significant) \\
\hline
\end{tabular}


Table 3 Secondary Outcomes Among Participants in the Severe Category

\begin{tabular}{|c|c|c|c|c|c|c|c|}
\hline & \multicolumn{2}{|l|}{ Severe A } & \multicolumn{2}{|l|}{ Severe B } & \multicolumn{2}{|l|}{ Severe C } & \multirow{2}{*}{$\begin{array}{l}\text { P-value } \\
\text { (Intergroups) }\end{array}$} \\
\hline & Number & $\%$ & Number & $\%$ & Number & $\%$ & \\
\hline \multicolumn{8}{|l|}{ Number of deaths during the study period } \\
\hline All-cause mortality & $6 / 21$ & 28.57 & $3 / 20$ & 15 & $5 / 22$ & 22.72 & $>0.05$ \\
\hline $\begin{array}{l}\text { Increase in oxygen requirement on Ist follow-up (at } \\
72 \text { hours) }\end{array}$ & $|5 / 2|$ & 71.42 & $17 / 20$ & 85 & $|8 / 2|$ & 85.71 & $>0.05$ \\
\hline $\begin{array}{l}\text { Increase in oxygen requirement on } 2 \text { nd follow-up } \\
\text { (at } 144 \text { hours) }\end{array}$ & $6 / 11$ & 54.54 & $9 / 13$ & 69.23 & $9 / 12$ & 75 & $>0.05$ \\
\hline \multicolumn{8}{|l|}{ Requirement of non-invasive ventilation (NIV) } \\
\hline NIV on Ist follow-up (at 72 hours) & $5 / 21$ & 23.8 & $2 / 20$ & 10 & $5 / 21$ & 23.8 & $>0.05$ \\
\hline NIV on 2nd follow-up (144 hours) & $3 / 9$ & 33.33 & $\mathrm{I} / \mathrm{I}$ & 9.09 & $1 / 6$ & 16.66 & $>0.05$ \\
\hline \multicolumn{8}{|l|}{ Requirement of mechanical ventilation (MV) } \\
\hline MV on Ist follow-up (at 72 hours) & $2 / 21$ & 9.52 & $1 / 20$ & 5 & $5 / 21$ & 23.8 & $>0.05$ \\
\hline MV on 2nd follow-up (144 hours) & $3 / 11$ & 27.27 & $1 / 13$ & 7.69 & $1 / 12$ & 8.33 & $>0.05$ \\
\hline Mean duration of hospital stays & \multicolumn{2}{|l|}{$\begin{array}{l}14.08 \text { days } \\
(S D=\mid 1.35)\end{array}$} & \multicolumn{2}{|l|}{$\begin{array}{l}14.15 \text { days } \\
(S D=6.08)\end{array}$} & \multicolumn{2}{|l|}{$\begin{array}{l}\mid 4.42 \text { days } \\
(S D=\mid 3.27)\end{array}$} & $>0.05$ \\
\hline
\end{tabular}

Notes: Participants who are already on NIV/MV at the time of allocation were excluded in determining the proportion.

Abbreviation: SD, standard deviation.

have been too small to evaluate that subgroup. ${ }^{17}$ However, treatment by severe group $\mathrm{C}$ is very ineffective. Recently the recovery trial proves no benefit of the lopinavir+ritonavir combination. ${ }^{14}$ Though ribavirin was added to increase the effectiveness, triple antiviral combinations could not succeed in contrast to HCQ+ribavirin.

An amendment was also made in the trial to reduce the doses of ribavirin due to the development of deranged liver enzymes; when this change was implemented six patients had been enrolled in the trial, but only two had received ribavirin in two groups. However, this has given a good outcome that ribavirin should not be used with $1,800 \mathrm{mg}$ loading dose and 1,200 mg BD maintenance dose in the Indian population, where it could lead to druginduced liver injury. This trial establishes lesser ribavirin doses for Indians compared to the western population if the replication of the study is intended in the near future. ${ }^{16}$ When we compare the study results between the participants receiving the non-standard group of treatments with that of remdesivir, a similar observation is made on the aspects of clinical recovery of symptoms and laboratory abnormalities, need of oxygen/ventilator requirements without any mortality benefit as in the remdesivir study. However, the results are not backed by a statistically significant outcome to advocate for a strong consideration on the use of this antiviral combination therapy.

This trial has several limitations posed upon by a relatively small sample size. It did not complete full enrolment owing to the development of new studies, suggesting Remdesivir to have beneficial effects in $\mathrm{O}_{2}$ requiring patients and also more patients opting for newer agents during the trial period, which becomes a major factor in premature discontinuation of the study for those participants. ${ }^{21}$ Our study was also non-blinded and lacks heterogeneity of different populations being a singlecenter study. With emerging data suggesting COVID-19 to have a more protracted course, different outcomes may have been missed with the limited follow-up period. There are also emerging strains of new COVID-19 with variable degrees of disease manifestations in regards to severity and organ involvement. Hence, concern also arises about the efficacy of these drugs in those newer strains and hence needs further evaluation.

The trial was also not devoid of challenges as it was conducted during a time of restricted travel and restricted entry of nonessential personnel. Monitoring visits often were performed remotely and strict implementation of isolation and other measures of infectious control was 
a major hindrance in drawing and processing samples in defined periods. There was also a shortage of trial-related supplies in between the study period such as reagents for the inflammatory markers. There was also the publication of results by the WHO from the Solidarity Trial in July 2020, which recommended discontinuation of HCQ and lopinavir/ritonavir arms, which resulted in a decrease in further enrolment of participants of the study. ${ }^{22}$

\section{Conclusion}

Standard therapy is advocated for the non-severe category of patients. However, based on the observations in the severe symptomatic group, the antiviral combination therapy mainly $\mathrm{HCQ}+$ ribavirin may have a role in improving clinical recovery in patients with COVID-19 and reducing the period of morbidity. For safety concerns in India, the dose of ribavirin should be halved compared to doses used in western countries. Forward research on a variety of therapeutic approaches including novel antivirals, modifiers of the immune response, or other intrinsic pathways is still the need of the hour.

\section{Data Sharing Statement}

The authors intend to share individual deidentified participant data of all studied variables after contacting the corresponding author's email address and these data will be available for 10 years.

\section{Ethics Statement}

All participants were informed about the purpose of the trial, consented, and the trial was conducted in accordance with the Declaration of Helsinki.

\section{Acknowledgments}

Thanks to Prof Manoj Gupta, Prof UB Mishra of the institute for providing logistics required for the trial; to Dr. Arkapal Bandyopadhyay, Dr Ramanuj Samanta, Dr Rohit Walia, Dr Itish Patnaik, Dr Gaurav Chikara, Dr Ravi Gupta, and Dr Bharat Bhusan Bhardwaj, for helping protocol preparation and handling drug complications in any of the study participants; and to Dr Deepjyoti Kalita and Dr Puneet Gupta for performing laboratory diagnosis of the study participants.

\section{Author Contributions}

All authors made substantial contributions to the conception and design, acquisition of data, or analysis and interpretation of data; took part in drafting the article or revising it critically for important intellectual content; agreed to submit to the current journal; gave final approval of the version to be published; and agree to be accountable for all aspects of the work.

\section{Disclosure}

The authors report no conflicts of interest in this work.

\section{References}

1. Centers for Disease Control and Prevention. Evidence used to update the list of underlying medical conditions that increase a person's risk of severe illness from COVID-19. US Dept Health Hum Serv. 2020;2019:1-6.

2. Banerjee D, Popoola J, Shah S, Ster IC, Quan V, Phanish M. COVID19 infection in kidney transplant recipients. Kidney Int. 2020;97 (6):1076-1082. doi:10.1016/j.kint.2020.03.018

3. Zambrano LD, Ellington S, Strid P, et al. Update: characteristics of symptomatic women of reproductive age with laboratory-confirmed SARS-CoV-2 infection by pregnancy status - United States, January 22-October 3, 2020. MMWR Morb Mortal Wkly Rep. 2020;69(44):1641-1647. doi:10.15585/mmwr.mm6944e3

4. Whittaker A, Anson M, Harky A. Neurological manifestations of COVID-19: a systematic review and current update. Acta Neurol Scand. 2020;142(1):14-22. doi:10.1111/ane.13266

5. Henry BM, de Oliveira MHS, Benoit S, Plebani M, Lippi G. Hematologic, biochemical and immune biomarker abnormalities associated with severe illness and mortality in coronavirus disease 2019 (COVID-19): a meta-analysis. Clin Chem Lab Med. 2020;58 (7):1021-1028. doi:10.1515/cclm-2020-0369

6. Agarwal A, Chen A, Ravindran N, To C, Thuluvath PJ. Gastrointestinal and liver manifestations of COVID-19. J Clin Exp Hepatol. 2020;10(3):263-265. doi:10.1016/j.jceh.2020.03.001

7. Sachdeva M, Gianotti R, Shah M, et al. Cutaneous manifestations of COVID-19: report of three cases and a review of literature. $J$ Dermatol Sci. 2020;98(2):75-81. doi:10.1016/j.jdermsci.2020.04.011

8. Liu PP, Blet A, Smyth D, Li H. The science underlying COVID-19: implications for the cardiovascular system. Circulation. 2020;142 (1):68-78. doi:10.1161/CIRCULATIONAHA.120.047549

9. Bikdeli B, Madhavan MV, Jimenez D, et al. COVID-19 and thrombotic or thromboembolic disease: implications for prevention, antithrombotic therapy, and follow-up: JACC state-of-the-art review. J Am Coll Cardiol. 2020;75(23):2950-2973. doi:10.1016/j.jacc.2020.04.031

10. Liu J, Cao R, Xu M, et al. Hydroxychloroquine, a less toxic derivative of chloroquine, is effective in inhibiting SARS-CoV-2 infection in vitro. Cell Discov. 2020;6(1):16. doi:10.1038/s41421-020-0156-0

11. Indian Council of Medical Research [Internet]. Advisory on the use of hydroxychloroquine as prophylaxis for SARS-CoV-2 infection [Internet]; 2020:1-3. Available from: https://www.mohfw.gov.in/pdf/ AdvisoryontheuseofHydroxychloroquinasprophylaxisforSARSCoV2i nfection.pdf. Accessed September 7, 2021.

12. Zhou D, Dai S-M, Tong Q. COVID-19: a recommendation to examine the effect of hydroxychloroquine in preventing infection and progression. $J$ Antimicrob Chemother. 2020;75(7):1667-1670. doi:10.1093/jac/dkaa114

13. Gao J, Tian Z, Yang X. Breakthrough: chloroquine phosphate has shown apparent efficacy in treatment of COVID-19 associated pneumonia in clinical studies. Biosci Trends. 2020;14(1):72-73. doi:10.5582/bst.2020.01047

14. Horby PW, Mafham M, Bell JL; RECOVERY Collaborative Group. Lopinavir-ritonavir in patients admitted to hospital with COVID-19 (RECOVERY): a randomised, controlled, open-label, platform trial. Lancet. 2020;396(10259):1345-1352. doi:10.1016/S0140-6736(20) 32013-4 
15. Cao B, Wang Y, Wen D, et al. a trial of lopinavir-ritonavir in adults hospitalized with severe covid-19. $N$ Engl J Med. 2020;382 (19):1787-1799. doi:10.1056/NEJMoa2001282

16. Khalili JS, Zhu H, Mak NSA, Yan Y, Zhu Y. Novel coronavirus treatment with ribavirin: groundwork for an evaluation concerning COVID-19. J Med Virol. 2020;92(7):740-746. doi:10.1002/ jmv. 25798

17. Sanders JM, Monogue ML, Jodlowski TZ, Cutrell JB. Pharmacologic treatments for Coronavirus Disease 2019 (COVID-19): a review. JAMA. 2020;323(18):1824-1836.

18. Kapoor M, Panda PK, Mohanty V. Pharmacotherapy for COVID-19: a ray of hope. IntechOpen. 2021. Available from:: https://www.inte chopen.com/online-first/pharmacotherapy-for-covid-19-a-ray-ofhope. Accessed September 7, 2021.
19. Lippi G, Wong J, Henry BM. Hypertension in patients with coronavirus disease 2019 (COVID-19): a pooled analysis. Pol Arch Intern Med. 2020;130(4):304-309.

20. COVID-19 Treatment Guidelines Panel. Coronavirus Disease 2019 (COVID-19) treatment guidelines. National Institutes of Health. Available from: https://www.covid19treatmentguidelines.nih.gov/. Accessed September 7, 2021.

21. The U.S. Food and Drug Administration. FDA approves first treatment for COVID-19; 2020. Available from: https://www.fda.gov/ news-events/press-announcements/fda-approves-first-treatment-covid -19. Accessed May 30, 2021.

22. WHO Solidarity Trial Consortium. Repurposed antiviral drugs for Covid-19 - interim WHO solidarity trial results. $N$ Engl $J$ Med. 2021;384(6):497-511. doi:10.1056/NEJMoa2023184

\section{Publish your work in this journal}

Clinical Pharmacology: Advances and Applications is an international, peer-reviewed, open access journal publishing original research, reports, reviews and commentaries on all areas of drug experience in humans. The manuscript management system is completely online and includes a very quick and fair peer-review system, which is all easy to use. Visit http://www.dovepress.com/testimonials.php to read real quotes from published authors.

Submit your manuscript here: https://www.dovepress.com/clinical-pharmacology-advances-and-applications-journal 